\title{
A RESEARCH REVIEW ON RETRIEVAL TECHNIQUES OF DIGITAL IMAGES
}

\author{
M. Muthuraman \\ Research Scholar and Assistant Professor, PG and Research Department of Computer Science, \\ H.H. The Rajah’s College (A), Pudukkottai, Tamilnadu- 622001 \\ Email:muthuraman70@gamil.com \\ Dr. S. Ravichandran \\ Assistant Professor and Head, PG and Research Department of Computer Science, \\ H.H. The Rajah’s College (A), Pudukkottai, Tamilnadu- 622001 \\ Email:rajahsravis@gmail.com
}

\begin{abstract}
Image Retrieval (IR) is an exciting and growing research areain multimedia. IR techniques are required in many fields of digital imaging like Medical Imaging, Remote sensing, Forensic Science, Military and printing. Images can be retrieved based on content (Content based image retrieval CBIR)and descriptive tags of images. This paper provides a review of current prevalent IR techniques in image retrieval.
\end{abstract}

\section{INTRODUCTION}

The continuous use of internet and handheld devices and availability of higher bandwidths allows people to create, store and explore multimedia content [1]. Multimedia content of various applications and social networking sites together contribute towards the creation of vast volume of multimedia content. This multimedia content, specifically images, can be used to obtain useful information. Image Retrieval is locating and retrieving images that satisfy a pre-defined need, from large image data stores. Image retrieval techniques, tools and algorithms originate from multiple fields like statistics, pattern recognition, signal processing and computer vision. Image retrieval needs efficiency and accuracy in searches, matches and retrievals[2]. Image retrieval (IR) techniques are attracting the attention of professionals from different industries [3] in addition to researchers. Efficiency, speed and accuracy are the driving forcesbehind image retrievals.Many image retrieval systems have been developed [4]. Two IR techniques are generally used namely Text-based and Content-based. Text Based IR techniques were used from 1970s, where textual annotations were searched for retrieving images. Images were described manually and ina database of images, it became a tedious and error prone job [5]. The text based method was subjective and incomplete and failed to specify image features. The approach slowly lost popularity due to inappropriate fetches of images based on notations. The second IR technique, Content Based image retrieval (CBIR) emerged in the 1990's to overcome drawbacks in Text Based IR techniques [6]. The technique uses unique image features like color, texture and shape, to access or retrieve them. These features can be easily derived from the image without external knowledge base [7]. Content-based implies searches analyze the actual image contents of the image other than the image's metadata and its utility can be proved in web based image searches, where the search engines rely purely on meta information and produce inappropriate detection [8]. Though images can be retrieved using texture, color or shape, color is the most important feature in IR. Colors are retrieved using color histograms or Color moments or Color correlograms. Histograms do not store spatial information. Color moments take into account the first four moments in calculation and measure color similarity between images, Color correlogram providethe probability of discovering color pairs at a specific pixel distance. Spatial information is provided in Color correlogram thus enabling better output than color histograms. The objective of this paper is to detail on a few existing and prevalent image retrieval algorithms and techniques.

\section{IMAGE RETRIEVAL FEATURES}

Image low level features like color, shape are analyzed to create vectors as its index and stored in a database for comparisons [9]. Any input query image features are extracted and matched with this stored image features for returning retrieval images. For efficient image retrieval appropriate feature vectors to represent image content must be created and stored. Conceptual model of content based image retrieval is depicted in Figure 1. 


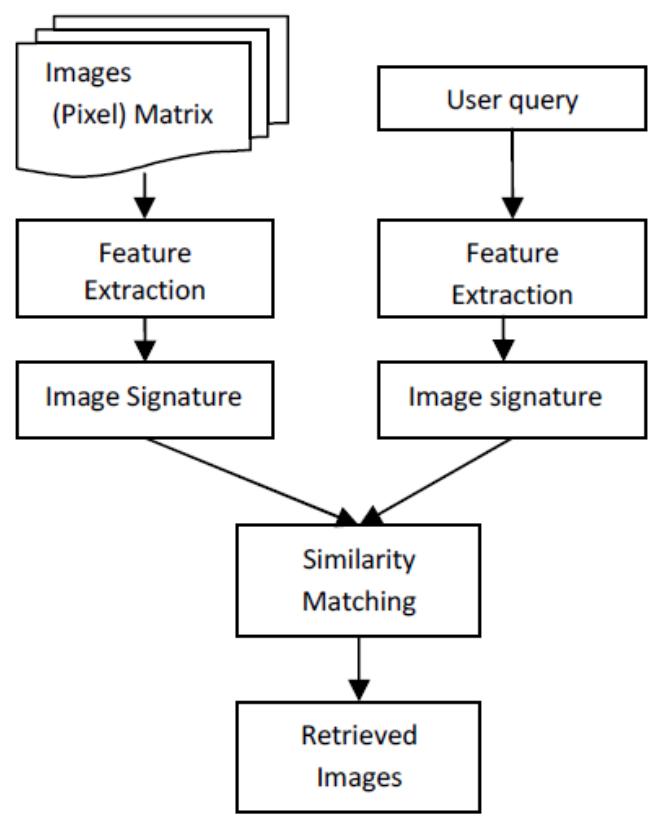

Figure 1 Content Based Image Retrieval

\subsection{Image Retrieval Using Extraction of Color}

Color in an image attracts the human eye, due the eye's sensitivity to colors. Any human eye can recognize most colored objects included in an image. Methods based on color similarity and its variations have been proposed is IR systems. Each database image is analyzed and its features computed. The Global Color Histogram (GCH) method represents images by their histograms. Similarities in images are determined by the distance between an image its color histogram. The drawback of this system is that it may not represent an image sufficiently and variations may occur due to variation in intensity, color distortions or cropping of images. The second method, Local Color Histograms (LCH), dividesimages and obtains a separate histogram for each block. The image histogram is a compilation of block histogram together. In comparisons, image blocks are compared and the distance is calculated as the sum of the distances between blocks. This method has been found to be more dependable as it enables image region comparisons [10]. Though, RGB colors are most commonly used, it is not suitable for descriptions practical to the naked eyes. Image processing algorithms use HSV (hue, saturation, value), since they are colors that are natural to the human eyes [11] and generate color histograms that outperformsystems based on RGB.

\subsection{Image Retrieval Using Extraction of Texture}

Texture in an image is important to human vision and can be used to identify and describe image contents like crops, mountains etc. Texture with color helps describe characteristics in a better way. If color is imagined as a point, then texture is a neighborhood of points. Image retrievals using texture in an image can based on edge histogram descriptor (EHD), gray-level co-occurrence matrix (GLCM) or wavelet moments. Recent methods introduced were BDIP (block difference of inverse probabilities) and BVLC (block variation of local correlation coefficients). Former is a non-linear operator with its local maximum normalized, while the latter is a highest difference between local correlations based on orientations normalized by local variance for measuring smoothness. They effectively measure local brightness and local texture smoothness. Both the methods are bounded and normalized to reduce illumination effects. The images are extracted as $2 * 2$ for measuring local image characteristics more closely [12]. Both operators were applied to ROI determination, image retrieval, face detection and texture classification and the results were found to be satisfactory.

\subsection{Image Retrievals using Extraction of Shape}

Though shape is an important visual feature in image, it is difficult to describe or represent shape. Three dimension images when cast into two dimensional images lose object information of one dimension. Thus, the shape projected in 2D is incomplete. Further complications occur due to defects, distortion and noise in images. Effective shape representation in Computer graphics or mathematics cannot be used in shape recognition. Shape features in an image can be extracted using contour or region based methods. Contours use boundary of an object to extract its shape, ignoring features inside the boundaries. Segmentation is applied first to an image in Region-based image retrieval methods. A threshold value is set for favorable results and edge detection methods are used for boundary extraction in an image. Shape are represented by polygonal approximation, B-splines, Fourier descriptors, deformable templates, invariant moments and curvature scale space [13]. 


\section{PREVIOUS STUDIES IN IR}

Simardeep Kaur et al. [14] presented HSV based color space IR method, based on the color distribution of the images. The retrieval HSV values has a high recall and precision of retrieval. S. P. James [15] presented an image retrieval system using HSV color features to retrieve facial images. Iyad Aldasouqi and Mahmoud Hassan [16], detected human faces in color images using HSV color model and could be used some real-time applications. Shamik Sural et al. [17]analyzed the properties of the HSV with emphasis on the variation in HS Intensity values of an image pixel. It was applied for image segmentation and histogram generation applications. Young Deok et al. [18] proposed an image retrieval method based on the combination of BDIP and BVLC with better performance in precision. Yu-Len Huang et al. [19] classified benign and malignant breast tumors on medical ultrasound with high precision by using texture features in a computer-aided diagnosis (CAD) systems. Ying Ai Ju et al. [20] used local statistics of gradients and correlations and used BDIP and BVLC types for face recognition. Their proposed method yielded good results. Most techniques in shape extraction used whole shape matching. Fourier descriptor were found to be efficient, but were not suitable for partial shape matching. B. Ramamurthy [21] proposed a novel approach for medical image retrieval based on shape feature using edge detection algorithm for extraction of image shape and K-means for extraction of different regions of the image.

\section{IMAGE RETRIEVAL TECHNIQUES}

A few IR techniques are detailed below.

- Relevance Feedback: It is a technique where Machine provides the initial image retrieval outcomes. The user then provides a feedback on the retrieved image's relevance and the search continues based on the user feedback.

- Semantic Template: In this method a high level image retrieval is supported. It is rarely used. It is a representative feature calculated from sample images.

- Wavelet Transform: It is based on tiny waves with limited duration and variable frequency. Discrete Wavelet transform frequency of images into 4 parts namely higher frequency (HH), High Low Frequency (HL), Low High Frequency (LH), and Lower frequency (LL). Moments for each of these four parts are calculated and stored for comparison of similar images.

- Gabor Filter: It is used for texture analysis and uses a two dimensional Gabor function $\mathrm{g}(\mathrm{x}, \mathrm{y})$ consisting of sinusoidal plane wave of frequency and orientation, while a Gaussian Envelope modulates it.

4.1 IR Systems

Many content based image retrieval systems have been developed and some of them are detailed below

- QBIC: developed by IBM, retrieves color or texture or shape from images. Users can enter a query image or sketch. QBIC uses an $\mathrm{R}^{*}$-tree index to sort images based on significance.

- Virage: developed by Virage, retrieves global color, local color, texture and shapes. Users can enter query image or sketch or selected color or selected texture

- Photobook: developed by MIT, Massachusetts, retrieves by computing shape, texture and required features. It is used in image texture retrieval, human faces and shapes

- Chabot: retrieves an image by using a combination of color and text features of images.

- VisualSEEk: developed by Columbia University, retrieves based on shape, region color, spatial location and keywords. Results are refined with relevant feedback

- MARS: developed by University of Illinois, uses many features to identify objects, similarity measures to compare with stored objects. Uses relevant feedback and user feedback adjusts the feature weights.

- Netra: incorporates image segmentation techniques to retrieve region based objects and uses texture, color, shape and spatial location.

\section{CONCLUSION}

This paper has detailed on past and current technical achievements in image retrieval systems. The survey of image features that can be used for image retrievals have been discussed in this paper. IR process starts on a user query for images in the form of text, image or a string. IR content retrieval is based on color, texture or shape of the query image which is then matched with images in the image database. Color is invariant to image size and orientation and a very useful feature in image retrievals. Texture based IR techniques are also used, but give maximum output and accuracy when integrated with color features of images in retrievals. Object shapes provide information that can be recognized by humans. Many researches have focused on using a single feature. Ii is difficult to find more accuracy in image retrievals using only one feature of an image. New image annotation techniques can be for semantic based IR. Text mining techniques can be combined with visual-based techniques in IR. Many prevailing techniques have been detailed with details on image features and possible future direction in Image Retrieval Techniques. This paper concludes that a hybrid or combination of image 
features, if used by image retrieval techniques, the results will be better than existing techniques and thus lay for the future development in IR techniques.

\section{REFERENCES}

[1] Swapnalini Pattanaik ,D.G. Bhalke "Efficient Content based Image Retrieval System using Mpeg-7 Features”, International Journal of Computer Applications (0975 - 8887) Volume 53- No.5, September 2012.

[2] Xiaoling, W. (2009), "A Novel Circular Ring Histogram for Content-Based Image Retrieval”, First International Workshop on Education Technology and Computer Science, Vol. 2,Pp.785-788.

[3] Sang-Mi Lee, Hee-Jung Bae and Sung-Hwan Jung, "Efficient Content based Image Retrieval Methods using Color \& Texture," ETRI Journal, Vol. 20, No. 3, pp.272-283, 1998.

[4] D. D. Dhobale, B. S. Patil, S. B. Patil, V. R. Ghorpade (2011) Semantic understanding of Image content International Journal of Computer Science Issues, Vol. 8, Issue 3, No. 2, 1694-0814.

[5] Aman Chadha, Sushmit Mallik and Ravdeep Johar (2012) Comparative Study and Optimization of Feature-Extraction Techniques for Content based Image Retrieval” IJCA (0975 - 8887) Volume 52- No.20

[6] Changhu Wang, Lei Zhang and Hong-Jiang Zhang (2008) Learning to Reduce the Semantic Gap in Web Image Retrieval and Annotation SIGIR'08

[7] D. D. Dhobale, B. S. Patil, S. B. Patil, V. R. Ghorpade (2011) Semantic understanding of Image content International Journal of Computer Science Issues, Vol. 8, Issue 3, No. 2, 1694-0814.

[8] Dr. H.B.Kekre, Sudeep D. Thepade and Akshay Maloo, "Image Retrieval using Fractional Coefficients of Transformed Image using DCT and Walsh Transform,” International Journal of Engineering Science and Technology. Vol. 2, No. 4, pp. 362-371, 2010.

[9] J. Kang and W. Zhang, "A framework for image retrieval with hybrid features”, Control and Decision Conference (CCDC), 2012 24th Chinese, IEEE, (2012), pp. 1326-1330.

[10] A. Afifi, "Image Retrieval Based on Content Using Color Feature”, (Doctoral dissertation, Master dissertation, Computer Engineering Department, Islamic University of Gaza, Palestine), (2011).

[11] R. C. Gonzalez, R. E. Woods and S. L. Eddins, "Digital image processing using MATLAB”, 2, Knoxville: Gatesmark Publishing, (2009).

[12] G. M. Dahane and S. Vishwakarma, “Content Based Image Retrieval System”, IJEIT, 1, (2012), pp. 92-96.

[13] J. Vogel and B. Schiele, "Performance evaluation and optimization for content-based image retrieval”, Pattern Recognition, vol. 39, no. 5, (2006), pp. 897-909.

[14] S. Kaur and V. K. Banga, "Content Based Image Retrieval: Survey and Comparison between RGB and HSV”, model AMRITSAR COLLEGE OF ENGG \& TECHNOLOGY, Amritsar, India.

[15] "Face Image Retrieval with HSV Color Space using Clustering Techniques" Samuel Peter James Assistant Professor, Department of Computer Science and Engineering, Chandy College of Engineering, Thoothukudi, Tamil nadu, INDIA. EMail:i.samuelpeterjames@gmail.com.

[16] I. Aldasouqi and M. Hassan, "Human face detection system using HSV", Proc. Of 9th WSEAS Int. Conf. on Circuits, Systems, Electronics, Control \& Signal Processing (CSECS’10). Atenas, Grecia, (2010).

[17] S. Sural, Q. Gang and S. Pramanik, "Segmentation and histogram generation using the HSV color space for image retrieval”, Image Processing. 2002. Proceedings. 2002 International Conference on. vol. 2, IEEE, (2002).

[18] Y. D. Chun, S. Y. Seo, and N. C. Kim, "Image retrieval using BDIP and BVLC moments", Circuits and Systems for Video Technology, IEEE Transactions on vol. 13, no. 9, (2003), pp. 951-957.

[19] Y.-L. Huang, K.-L. Wang and D.-R. Chen, "Diagnosis of breast tumors with ultrasonic texture analysis using support vector machines', Neural Computing \& Applications, vol. 15, no. 2, (2006), pp. 164-169.

[20] Y. A. Ju, "Face recognition using local statistics of gradients and correlations”, Proc. European Signal Processing Conf. (2010).

[21] B. Ramamurthy and K. R. Chandran, "CBMIR: shape-based image retrieval using canny edge detection and k-means clustering algorithms for medical images”, International Journal of Engineering Science and Technology, vol. 3, no. 3, (2011), pp. 209-212. 